\title{
Bivariate Polynomial Interpolation at the Geronimus Nodes
}

\author{
Lawrence A. Harris
}

\begin{abstract}
We consider a class of orthogonal polynomials that satisfy a threeterm recurrence formula with constant coefficients. This class contains the Geronimus class and, in particular, all four kinds of the Chebyshev polynomials. There are alternation points for each of these orthogonal polynomials that have a special compatibility with the polynomials of lower index. These points are the coordinates of two sets of nodes in $\mathbb{R}^{2}$, which we call Geronimus nodes. The Chebyshev nodes considered separately by Yuan Xu and the author are a special case.

We obtain an explicit formula (involving the reproducing kernel) for bivariate Lagrange polynomials for the Geronimus nodes and we apply this to obtain a bivariate interpolation theorem and a cubature formula. These theorems are a consequence of a surprisingly elementary connection between Lagrange polynomials, interpolation formulas and cubature formulas, which we explain in an appendix. Finally, we discuss a general bivariate Markov theorem where polynomials in the Geronimus class are extremal.
\end{abstract}

\section{A class of orthogonal polynomials}

Given real constants $a, b, c$ and $d$, let $\left\{p_{n}\right\}$ be the sequence of polynomials defined recursively by

$$
\begin{gathered}
p_{0}(x)=1, \quad p_{1}(x)=a x+b, \\
p_{n+1}(x)=(c x+d) p_{n}(x)-p_{n-1}(x), \quad n \geq 1 .
\end{gathered}
$$

It follows from Favard's Theorem [4, Th. 4.4] that if $a c>0$ then $\left\{p_{n}\right\}$ is a sequence of orthogonal polynomials with respect to a positive definite moment functional $\ell$ satisfying

$$
\ell(1)=\frac{c}{a}, \quad \ell\left(p_{n}^{2}\right)=1, \quad n \geq 1 .
$$

This functional gives the moments of a compactly supported positive Borel measure $\mu$ which can have up to two atoms. (See [5] and [16] for an explicit expression.) We shall always assume that $a>0$ and $c>0$. (If both $a$ and $c$ are negative, consider instead $\tilde{p}_{n}(x)=p_{n}(-x)$.)

2010 Mathematics Subject Classification. Primary 65D05; Secondary 65D32, 42C05. 
Our discussion can be adapted to the more general case where there are real constants $\alpha, \beta, \gamma, \delta, \lambda$ and $\mu$ with $\mu>0$ and $\alpha \gamma \lambda>0$ and $q_{n}$ is a sequence of polynomials satisfying

$$
\begin{gathered}
q_{0}(x)=\lambda, \quad q_{1}(x)=\alpha x+\beta, \\
q_{n+1}(x)=(\gamma x+\delta) q_{n}(x)-\mu q_{n-1}(x), \quad n \geq 1,
\end{gathered}
$$

since $q_{n}=\lambda \mu^{n / 2} p_{n}$ where $p_{n}$ satisfies (1.1). On the other hand, one can reduce to the case $c=2$ and $d=0$. Specifically, if $p_{n}$ is a sequence of polynomials satisfying (1.1) and if $\alpha=2 a / c$ and $\beta=b-a d / c$, then

$$
p_{n}(x)=g_{n}\left(\frac{c x+d}{2}\right)
$$

where

$$
\begin{gathered}
g_{0}(x)=1, \quad g_{1}(x)=\alpha x+\beta, \\
g_{n+1}(x)=2 x g_{n}(x)-g_{n-1}(x), \quad n \geq 1 .
\end{gathered}
$$

The class (1.5) has been considered by Geronimus [9] (see also [15]) and is a special case of the $q$-Racah polynomials $[\mathbf{2}$, p. 27-28] by formula (1.6) below. See [17] for the case where polynomials are normalized to monic polynomials.

We single out four special cases of the sequence $p_{n}$ of polynomials satisfying (1.1) for fixed $c$ and $d$. Put $t_{n}=p_{n}$ when $a=c / 2, b=d / 2$, put $u_{n}=p_{n}$ when $a=c, b=d$, put $v_{n}=p_{n}$ when $a=c, b=d-1$, and put $w_{n}=p_{n}$ when $a=c$, $b=d+1$. In these cases, equation (1.4) holds where $g_{n}$ is the Chebyshev polynomial of the corresponding kind. These are given in Table 1 below where $c=2, d=0$ and $k_{n}=\frac{\left(2^{n} n !\right)^{2}}{(2 n) !}$. (See $[\mathbf{1 4}]$ for further details.)

Table 1: The four kinds of Chebyshev polynomials

\begin{tabular}{l|l|l|l} 
Kind & Constants & Definition & Jacobi polynomial \\
\hline 1st & $a=1, b=0$ & $T_{n}(\cos \theta)=\cos n \theta$ & $T_{n}=k_{n} P_{n}^{(-1 / 2,-1 / 2)}$ \\
2nd & $a=2, b=0$ & $U_{n}(\cos \theta)=\frac{\sin (n+1) \theta}{\sin \theta}$ & $U_{n}=\frac{n+1}{2 n+1} k_{n} P_{n}^{(1 / 2,1 / 2)}$ \\
3rd & $a=2, b=-1$ & $V_{n}(\cos \theta)=\frac{\cos (n+1 / 2) \theta}{\cos (\theta / 2)}$ & $V_{n}=k_{n} P_{n}^{(-1 / 2,1 / 2)}$ \\
4th & $a=2, b=1$ & $W_{n}(\cos \theta)=\frac{\sin (n+1 / 2) \theta}{\sin (\theta / 2)}$ & $W_{n}=k_{n} P_{n}^{(1 / 2,-1 / 2)}$
\end{tabular}

For each of the kinds of the Chebyshev polynomials, the moment functional of (1.2) is given by a weight function $w(x)$ with support $[-1,1]$ and $d \mu(x)=w(x) d x$. These are given in Table 2 in the next section. Other examples are given in [4, p. 204-205].

The expected identities

$$
2 t_{n}=u_{n}-u_{n-2}, \quad v_{n}=u_{n}-u_{n-1}, \quad w_{n}=u_{n}+u_{n-1}
$$

follow by induction for $n \geq 1$, where we take $u_{-1}=0$. It is easy to show by backward induction on $j$ that

$$
p_{n}=p_{j} u_{n-j}-p_{j-1} u_{n-j-1}, \quad 1 \leq j \leq n
$$

since this equation is obviously true when $j=n$ and (1.1) with $n=j-1$ shows that it is true for $j-1$ when it is true for $j$ and $j \geq 2$. In particular, when $j=1$,

$$
p_{n}(x)=(a x+b) u_{n-1}(x)-u_{n-2}(x), \quad n \geq 1
$$


and it follows that

$$
p_{n}=\frac{a}{c} u_{n}+\left(b-\frac{a d}{c}\right) u_{n-1}+\left(\frac{a}{c}-1\right) u_{n-2}, \quad n \geq 1 .
$$

In the next section, we will apply the identities

$$
\begin{aligned}
p_{n+j}+p_{n-j} & =2 p_{n} t_{j}, \quad 0 \leq j \leq n, \\
p_{n+j}-p_{n-j} & =\left(p_{n+1}-p_{n-1}\right) u_{j-1}, \quad 0 \leq j \leq n, \\
p_{n+j}+p_{n-j-1} & =\left(p_{n}+p_{n-1}\right) v_{j}, \quad 0 \leq j \leq n-1, \\
p_{n+j}-p_{n-j-1} & =\left(p_{n}-p_{n-1}\right) w_{j}, \quad 0 \leq j \leq n-1,
\end{aligned}
$$

which are easily proved. For example, to prove (1.9), let $q_{j}=p_{n+j}+p_{n-j-1}$ and note that

$$
q_{j}(x)=(c x+d) q_{j-1}(x)-q_{j-2}(x), \quad 2 \leq j \leq n-1 .
$$

Thus, by induction, it suffices to show that $q_{j}=q_{0} v_{j}$ holds for $j=0$ and $j=1$. When $j=0$ this is clear and when $j=1$ we can obtain this by adding the identities

$$
\begin{aligned}
& p_{n+1}(x)=(c x+d) p_{n}(x)-p_{n-1}(x) \\
& p_{n-2}(x)=(c x+d) p_{n-1}(x)-p_{n}(x)
\end{aligned}
$$

The proofs of the other identities are similar.

If desired, one can remove the restrictions on the subscripts in the above identities for $p_{n}$ by adding the equation $p_{-n}=2 t_{n}-p_{n}$ to the definition of $p_{n}$ in (1.1).

For purposes of comparison, we observe that the generating function for the polynomials $p_{n}$ is given by

$$
\frac{1+[(a x+b)-(c x+d)] z}{z^{2}-(c x+d) z+1}=\sum_{n=0}^{\infty} p_{n}(x) z^{n}, \quad|c x+d| \leq 2,|z|<1 .
$$

The moments $\mu_{n}=\ell\left((c x+d)^{n}\right)$ can be computed recursively from the identities

$$
\begin{gathered}
\mu_{0}=E, \quad \mu_{1}=F \mu_{0}, \\
\mu_{n}=F \mu_{n-1}+E \sum_{k=1}^{[n / 2]} \frac{1}{k}\left(\begin{array}{c}
2(k-1) \\
k-1
\end{array}\right) \mu_{n-2 k}, \quad n \geq 2,
\end{gathered}
$$

where $E=c / a, F=(a d-b c) / a$ and $\left(\begin{array}{l}0 \\ 0\end{array}\right)=1$. If $p$ is any polynomial, the value of $\ell(p)$ can be obtained from this and the Taylor expansion for $p$ at $-d / c$. When $F=0$, we have

$$
\begin{aligned}
\mu_{2 k-1} & =0 \\
\mu_{2 k} & =\mu_{0} \sum_{j=0}^{k-1}\left[\left(\begin{array}{c}
k+j-1 \\
j
\end{array}\right)-\left(\begin{array}{c}
k+j-1 \\
j-1
\end{array}\right)\right] E^{k-j}
\end{aligned}
$$

for $k \geq 1$, where $\left(\begin{array}{c}n \\ -1\end{array}\right)=0$. 


\section{Alternation points for the Geronimus polynomials}

For a given $m \geq 1$, we show that the polynomial $p_{m}$ has $m+1$ alternation points $h_{0}, \ldots, h_{m}$ that satisfy a critical compatibility condition with polynomials of lower index. In particular, $p_{m}\left(h_{n}\right)=(-1)^{n}$ for $n=0, \ldots, m$.

TheOREM 2.1. For each positive integer $m$ there exist unique numbers

$$
\begin{aligned}
h_{0} & >h_{1}>\cdots>h_{m} \text { such that } \\
p_{m-j}\left(h_{n}\right) & =(-1)^{n} p_{j}\left(h_{n}\right), \quad n, j=0, \ldots, m .
\end{aligned}
$$

Proof. Let $\left\{x_{k, j}\right\}_{j=1}^{k}$ denote the $k$ distinct real roots of $p_{k}$ in decreasing order. Note that the leading coefficient of $p_{k}$ is positive since $a>0$ and $c>0$. We first consider the case where $m=2 k-1$. By the separation property [4, Theorem 5.3],

$$
x_{k-1, j}<x_{k, j}<x_{k-1, j-1}, \quad j=1, \ldots, k,
$$

where $x_{k-1,0}=\infty$ and $x_{k-1, k}=-\infty$. Define $q_{k}=p_{k}+\epsilon p_{k-1}$. If $\epsilon=1$ then $q_{k}\left(x_{k-1, j}\right)$ and $q_{k}\left(x_{k, j}\right)$ have opposite signs so there exists a number $y_{j}^{-}$with $x_{k-1, j}<y_{j}^{-}<x_{k, j}$ and $y_{j}^{-}$is a root of $p_{k}+p_{k-1}$. If $\epsilon=-1$ then $q_{k}\left(x_{k, j}\right)$ and $q_{k}\left(x_{k-1, j-1}\right)$ have opposite signs so there exists a number $y_{j}^{+}$with $x_{k, j}<y_{j}^{+}<$ $x_{k-1, j-1}$ and $y_{j}^{+}$is a root of $p_{k}-p_{k-1}$.

It follows from (1.9) and (1.10) with $n=k$ and $j$ replaced by $k-1-j$ that $p_{m-j}+p_{j}$ and $p_{m-j}-p_{j}$ are polynomial multiples of $p_{k}+p_{k-1}$ and $p_{k}-p_{k-1}$, respectively, for $j=0, \ldots, k-1$. Thus we may take $\left\{h_{n}\right\}$ to be the sequence $y_{1}^{+}, y_{1}^{-}, \ldots, y_{k}^{+}, y_{k}^{-}$.

Now suppose $m=2 k$. It follows from the classical Christoffel-Darboux formula $[4$, p. 24] that

$$
\begin{aligned}
& p_{k+1}^{\prime} p_{k}-p_{k}^{\prime} p_{k+1}>0, \\
& p_{k}^{\prime} p_{k-1}-p_{k-1}^{\prime} p_{k}>0,
\end{aligned}
$$

and adding these we obtain

$$
q_{k+1}^{\prime} p_{k}-p_{k}^{\prime} q_{k+1}>0
$$

where $q_{k+1}=p_{k+1}-p_{k-1}$. Let $\left\{y_{j}^{-}\right\}_{j=1}^{k}$ be the $k$ distinct real roots of $p_{k}$ in decreasing order and note that the sign of $p_{k}^{\prime}\left(y_{j}^{-}\right)$is $(-1)^{j-1}$ for each $j$. It follows from (2.2) evaluated at $y_{j}^{-}$that the sign of $q_{k+1}\left(y_{j}^{-}\right)$is $(-1)^{j}$. Also, $q_{k+1}\left(y_{1}^{-}\right)$and $q_{k+1}(y)$ have opposite signs for large $y$ and $q_{k+1}\left(y_{k}^{-}\right)$and $q_{k+1}(y)$ have opposite signs for large $-y$. Hence $q_{k+1}$ has $k+1$ distinct real roots $\left\{y_{j}^{+}\right\}_{j=1}^{k+1}$ satisfying

$$
y_{j+1}^{+}<y_{j}^{-}<y_{j}^{+}, \quad j=1, \ldots, k .
$$

It follows from (1.7) and (1.8) that $p_{m-j}+p_{j}$ is a polynomial multiple of $p_{k}$ for $j=0, \ldots, k$ and $p_{m-j}-p_{j}$ is a polynomial multiple of $p_{k+1}-p_{k-1}$ for $j=0, \ldots, k-1$. Thus we may take $\left\{h_{n}\right\}$ to be the sequence $y_{1}^{+}, y_{1}^{-}, \ldots, y_{k+1}^{+}$.

Uniqueness follows since in each case the sequence $h_{n}$ with $n$ even must be the sequence of roots $\left\{y_{j}^{+}\right\}$and the sequence $h_{n}$ with $n$ odd must be the sequence of roots $\left\{y_{j}^{-}\right\}$.

The Table 2 below gives the values of the points $h_{n}, 0 \leq n \leq m$, for each of the four kinds of Chebyshev polynomials. 
Table 2: Alternation points for the Chebyshev polynomials

\begin{tabular}{l|l|l|l} 
Kind & Weight on $(-1,1)$ & $h_{n}=\cos \theta_{n}$ & $\pi_{m}$ \\
\hline 1st & $w_{1}(x)=\frac{2}{\pi \sqrt{1-x^{2}}}$ & $\theta_{n}=\frac{n \pi}{m}$ & $\frac{1}{2}\left(T_{m+1}-T_{m-1}\right)$ \\
2nd & $w_{2}(x)=\frac{2}{\pi} \sqrt{1-x^{2}}$ & $\theta_{n}=\frac{(n+1) \pi}{m+2}$ & $U_{m+1}$ \\
3rd & $w_{3}(x)=\frac{1}{\pi} \sqrt{\frac{1+x}{1-x}}$ & $\theta_{n}=\frac{n \pi}{m+1}$ & $V_{m+1}-V_{m}$ \\
$4 \mathrm{th}$ & $w_{4}(x)=\frac{1}{\pi} \sqrt{\frac{1-x}{1+x}}$ & $\theta_{n}=\frac{(n+1) \pi}{m+1}$ & $W_{m+1}+W_{m}$
\end{tabular}

Note that by the proof of Theorem 2.1, the numbers $h_{n}$ in (2.1) are just the roots in decreasing order of the polynomial $\pi_{m}$ (of degree $m+1$ ) defined by

$$
\pi_{m}=\left\{\begin{array}{ll}
p_{k}^{2}-p_{k-1}^{2} & \text { if } m=2 k-1, \\
\left(p_{k+1}-p_{k-1}\right) p_{k} & \text { if } m=2 k
\end{array} .\right.
$$

It is not difficult to show that

$$
\pi_{m}(x)=(a x+b) p_{m}(x)-p_{m-1}(x)
$$

for all positive integers $m$. Indeed, when $m=2 k-1$, one can obtain (2.3) by applying the identity

$$
p_{k}(x)-p_{k-1}(x)=(a x+b) v_{k-1}(x)-v_{k-2}(x)
$$

in the equations (1.9) with $j=k-1$ and $j=k-2$, where $n=k$. When $m=2 k$, one can obtain (2.3) by applying the identity

$$
p_{k+1}(x)-p_{k-1}(x)=2\left[(a x+b) t_{k}(x)-t_{k-1}(x)\right]
$$

in the equations (1.7) with $j=k$ and $j=k-1$, where $n=k$. It is easy to deduce from (2.3) and (1.1) that

$$
\pi_{m}=\frac{a}{c} p_{m+1}+\left(b-\frac{a d}{c}\right) p_{m}+\left(\frac{a}{c}-1\right) p_{m-1} .
$$

The numbers $h_{n}$ of Theorem 2.1 are the points of evaluation in an $m+1$-point quadrature formula that has at most one more point than Gaussian quadrature and reduces to the Lobatto-Chebyshev formula $[\mathbf{7}, 2.7 .1 .14]$ when $w=w_{1}$. Let $\mathcal{P}_{n}(\mathbb{R})$ denote the space of all polynomials in a single variable with degree at most $n$.

THEOREM 2.2. Suppose the moment functional for the polynomials (1.1) with fixed $a, b, c, d$ is given by a nonnegative weight function $w(x)$ on $(-\infty, \infty)$. Then

$$
\int_{\mathbb{R}} p(x) w(x) d x=\sum_{n=0}^{m} \alpha_{n} p\left(h_{n}\right)
$$

for all $p \in \mathcal{P}_{2 m-1}(\mathbb{R})$, where

$$
\alpha_{n}=\frac{(-1)^{n} c}{\pi_{m}^{\prime}\left(h_{n}\right)}>0, \quad n=0, \ldots, m .
$$

This theorem applies, in particular, to each of the kinds of the Chebyshev polynomials where $w(t)$ and $h_{n}$ are as given in Table 2 and the weights are as given below.

1st) $\alpha_{n}=\frac{2}{m}$ for $0<n<m$ and $\alpha_{n}=\frac{1}{m}$ for $n=0, m$,

2nd) $\alpha_{n}=\frac{2\left(1-h_{n}^{2}\right)}{m+2}$ for $0 \leq n \leq m$, 
3rd) $\alpha_{n}=\frac{1+h_{n}}{m+1}$ for $0<n \leq m$ and $\alpha_{0}=\frac{1}{m+1}$,

4th) $\alpha_{n}=\frac{1-h_{n}}{m+1}$ for $0 \leq n<m$ and $\alpha_{m}=\frac{1}{m+1}$.

Note that when $w=w_{2}$, formula 2.5 is just the classical Gaussian quadrature and holds for all $p \in \mathcal{P}_{2 m+1}(\mathbb{R})$.

Proof of Theorem 2.2. The Lagrange polynomials for the points $\left\{h_{n}\right\}$ are given by

$$
P_{n}(x)=\frac{\pi_{m}(x)}{\pi_{m}^{\prime}\left(h_{n}\right)\left(x-h_{n}\right)}, \quad 0 \leq n \leq m .
$$

It follows from the classical Christoffel-Darboux formula [1, p. 261] and (2.3) that

SO

$$
K_{m-1}\left(x, h_{n}\right)=\frac{(-1)^{n}}{c}\left[\frac{\pi_{m}(x)}{x-h_{n}}-a p_{m}(x)\right]
$$

$$
P_{n}(x)=\alpha_{n} K_{m-1}\left(x, h_{n}\right)+\beta_{n} p_{m}(x)
$$

where

$$
\alpha_{n}=\frac{(-1)^{n} c}{\pi_{m}^{\prime}\left(h_{n}\right)}, \quad \beta_{n}=\frac{a}{\pi_{m}^{\prime}\left(h_{n}\right)} .
$$

The inequality $\alpha_{n}>0$ can be deduced from the identity

$$
\pi_{m}(x)=a^{2} c^{m-1} \prod_{n=0}^{m}\left(x-h_{n}\right), \quad m \geq 1 .
$$

Thus Theorem 2.2 follows from the 1-dimensional case of Theorem A-2 of the Appendix.

By (2.4), the polynomial $\pi_{m}$ is quasi-orthogonal of order 2 (or less) and thus is included in the discussion of quadrature given in [19].

\section{Interpolation at the Geronimus nodes}

The Geronimus nodes are finite sets of points in $\mathbb{R}^{2}$ associated with linear functionals on polynomials of two variables where the Geronimus polynomials in each of the variables are extremal. (See [10, Lemma 6].)

We define the even Geronimus nodes $\mathcal{N}_{0}$ to be the set of ordered pairs $\left(h_{n}, h_{q}\right)$, $0 \leq n, q \leq m$, where $n$ and $q$ are both even or both odd and the odd Geronimus nodes $\mathcal{N}_{1}$ to be the set of ordered pairs $\left(h_{n}, h_{q}\right), 0 \leq n, q \leq m$, where $n$ is even and $q$ is odd or $n$ is odd and $q$ is even. Thus if $k=0$ or $k=1$, then the Geronimus nodes are

where

$$
\mathcal{N}_{k}=\left\{\left(h_{n}, h_{q}\right):(n, q) \in Q_{k}\right\}
$$

$$
Q_{k}=\{(n, q): 0 \leq n, q \leq m, n-q=k \bmod 2\}
$$

For example, the Chebyshev nodes given in [10] are the Geronimus nodes for the case $a=1, b=0, c=2, d=0$ in (1.1). (See also [20].)

To define Lagrange polynomials for each set of Geronimus nodes, we first define

$$
\begin{aligned}
G_{m}(s, t, u, v)= & \frac{1}{2}\left[K_{m-1}(s, t, u, v)+K_{m}(s, t, u, v)\right] \\
& +\frac{a(a-c)}{2 c^{2}}\left[p_{m}(s) p_{m}(u)+p_{m}(t) p_{m}(v)\right]
\end{aligned}
$$


where

$$
K_{n}(s, t, u, v)=\sum_{i=0}^{n}{ }_{j=0}^{i}{ }^{\prime \prime} p_{i-j}(s) p_{j}(t) p_{i-j}(u) p_{j}(v)
$$

is the reproducing kernel for the inner product space of polynomials of degree at most $n$. (See (A-2) in the Appendix.) Here' means that the first term of the sum is multiplied by $a / c$ and " means that the first and last terms of the sum are multiplied by $a / c$ (but only once if these terms are the same). This convention allows us to treat the polynomials (1.1) as an orthonormal set in view of (1.2). (The function $G_{m}$ in (3.1) is one half of the function $G_{m}$ in [13] and one quarter of the function $G$ in $[\mathbf{1 1}]$.)

By (3.1), we can write

$$
\begin{aligned}
G_{m}(s, t, u, v)= & K_{m-1}(s, t, u, v)+\frac{1}{2} \sum_{j=1}^{m-1} p_{m-j}(s) p_{j}(t) p_{m-j}(u) p_{j}(v) \\
& +\frac{a^{2}}{2 c^{2}}\left[p_{m}(s) p_{m}(u)+p_{m}(t) p_{m}(v)\right]
\end{aligned}
$$

In particular, $G_{m}(s, t, s, t)>0$. Put $c_{n, q}=1 / G_{m}\left(h_{n}, h_{q}, h_{n}, h_{q}\right)$ and define

$$
P_{n, q}(s, t)=c_{n, q} G_{m}\left(s, t, h_{n}, h_{q}\right) .
$$

TheOREM 3.1. Let $k=0$ or $k=1$ and let $(n, q) \in Q_{k}$. Then $P_{n, q}$ is a polynomial of degree $m$ satisfying $P_{n, q}\left(h_{n}, h_{q}\right)=1$ and $P_{n, q}(x)=0$ for all $x \in \mathcal{N}_{k}$ with $x \neq\left(h_{n}, h_{q}\right)$.

Theorem 3.1 can be deduced easily from a bivariate Christoffel-Darboux identity that plays an important role in our proofs. To state this identity, let $\epsilon$ be an arbitrary real number and define

$$
\begin{aligned}
V_{j}(s, t) & =p_{m-j}(s) p_{j}(t)-\epsilon p_{j}(s) p_{m-j}(t), \quad j=0, \ldots, m, \\
W_{0}(s, t) & =(a s+b) p_{m}(s)-p_{m-1}(s)=\pi_{m}(s), \\
W_{j}(s, t) & =p_{m-j+1}(s) p_{j}(t)-\epsilon p_{j-1}(s) p_{m-j}(t), \quad j=1, \ldots, m .
\end{aligned}
$$

(Thus the symbols $V$ and $W$ from here on no longer denote the Chebyshev polynomials of the third and fourth kinds.) It follows from (2.3) and Theorem 2.1 that the even Geronimus nodes are common zeros of the polynomials (3.4)-(3.6) with $\epsilon=1$ and the odd Geronimus nodes are common zeros of these polynomials with $\epsilon=-1$. The Christoffel-Darboux identity

$$
\begin{aligned}
2 c(s-u) G_{m}(s, t, u, v)= & \sum_{j=0}^{m-1} '\left[V_{j}(s, t) p_{m-j-1}(u) p_{j}(v)-V_{j}(u, v) p_{m-j-1}(s) p_{j}(t)\right] \\
& +\sum_{j=0}^{m}{ }^{\prime \prime}\left[W_{j}(s, t) p_{m-j}(u) p_{j}(v)-W_{j}(u, v) p_{m-j}(s) p_{j}(t)\right]
\end{aligned}
$$


can be verified as in [11]. (An extra term is needed in [11] since $W_{0}$ is defined differently there.) Taking $\epsilon=0$ in this identity, we obtain

$$
\begin{aligned}
2 c G_{m}(s, t, u, v)= & \frac{1}{s-u} \sum_{j=0}^{m-1}\left[p_{m-j+1}(s) p_{m-j}(u)-p_{m-j+1}(u) p_{m-j}(s)+\right. \\
& \left.\quad p_{m-j}(s) p_{m-j-1}(u)-p_{m-j}(u) p_{m-j-1}(s)\right] p_{j}(t) p_{j}(v) \\
& +\frac{a(a-c)}{c} p_{m}(s) p_{m}(u)+\frac{a^{2}}{c} p_{m}(t) p_{m}(v) .
\end{aligned}
$$

The first statement of the following theorem is a consequence of Proposition A1 of the Appendix as in [10]. Let $\mathcal{P}_{n}\left(\mathbb{R}^{2}\right)$ denote the space of all real-valued polynomials in two variables with degree at most $n$.

Theorem 3.2. If $p \in \mathcal{P}_{m}\left(\mathbb{R}^{2}\right)$ and if $k=0$ or $k=1$ then there exists a linear combination $\bar{p}_{k}$ of $V_{1}, \ldots, V_{m}$ of (3.4) with $\epsilon=(-1)^{k}$ such that

$$
p=\sum_{(n, q) \in Q_{k}} p\left(h_{n}, h_{q}\right) P_{n, q}+\bar{p}_{k} .
$$

If $p$ has degree at most $m-1$ then (3.7) holds with $\bar{p}_{k}=0$.

In view of what we have shown, Theorem A-2 and Corollary A-3 of the Appendix apply to prove the second statement of the above theorem and the following cubature formula.

Theorem 3.3. Let $w(x)$ be as in Theorem 2.2 and let $k=0$ or $k=1$. Then

$$
\iint_{\mathbb{R}^{2}} p(s, t) w(s) w(t) d s d t=\sum_{(n, q) \in Q_{k}} c_{n, q} p\left(h_{n}, h_{q}\right)
$$

for all $p \in \mathcal{P}_{2 m-1}\left(\mathbb{R}^{2}\right)$.

Note that Theorem 3.3 applies, in particular, to each of the kinds of the Chebyshev polynomials where $w(x)$ and $h_{n}$ are as given in Table 2 .

Suppose $w$ is centrally symmetric, i.e., $w(-x)=w(x)$ for all $x \in \mathbb{R}$. By a theorem of Möller, the number $N$ of nodes of a cubature formula for the integral of (3.8) that holds for all $p \in \mathcal{P}_{2 m-1}\left(\mathbb{R}^{2}\right)$ satisfies

$$
N \geq\left(\begin{array}{c}
m+1 \\
2
\end{array}\right)+\left[\frac{m}{2}\right] .
$$

The number of elements in $Q_{k}$ has been counted in [10]. Thus the number of nodes in (3.8) is minimal when $m$ is even and $k=1$ and is at most one more than the minimal number of nodes otherwise.

Theorem 3.3 and a similar theorem of Bojanov and Petrova [3] combine to give a simpler formula for the coefficients $c_{n, q}$ in (3.3).

Theorem 3.4. $c_{n, q}=2 \alpha_{n} \alpha_{q}, \quad 0 \leq n, q \leq m$.

Proof. Define

$$
E(p)=\sum_{\text {even }} \alpha_{n} p\left(h_{n}\right), \quad F(p)=\sum_{\text {odd }} \alpha_{n} p\left(h_{n}\right),
$$


where the sums are taken over the even and odd integers $n$ from 0 to $m$, respectively. By Theorem 2.2,

$$
\begin{aligned}
E\left(p_{j}\right)+F\left(p_{j}\right) & =\int_{\mathbb{R}} p_{j}(x) w(x) d x=0, \quad 1 \leq j \leq 2 m-1, \\
E(1)+F(1) & =\int_{\mathbb{R}} w(x) d x=\ell(1), \\
E\left(p_{j}\right)-F\left(p_{j}\right) & =\int_{\mathbb{R}} p_{j}(x) p_{m}(x) w(x) d x=0, \quad 0 \leq j \leq m-1 .
\end{aligned}
$$

Hence, $E(1)=F(1)=\ell(1) / 2$ and $E\left(p_{j}\right)=F\left(p_{j}\right)=0$ for $1 \leq j \leq m-1$. Thus, in view of Theorem 2.2, Theorem 2.1 of $[\mathbf{3}]$ applies to show that

$$
\iint_{\mathbb{R}^{2}} p(s, t) w(s) w(t) d s d t=2 \sum_{(n, q) \in Q_{k}} \alpha_{n} \alpha_{q} p\left(h_{n}, h_{q}\right)
$$

for all $p \in \mathcal{P}_{2 m-1}\left(\mathbb{R}^{2}\right)$ and $k=0,1$. The required equality now follows from this and Theorem 3.3 with $p=P_{n, q}$.

\section{A general Markov theorem}

In this section we apply the interpolation results in the preceding section to obtain a general Markov theorem. Let $m \geq 1$ and $k \geq 0$. Define

$$
\alpha_{n, q}^{(k)}(r)=(-1)^{n} \hat{D}^{k} G_{n, q}(r, r)(1,-1)
$$

for $r \geq 0$, where $G_{n, q}(s, t)=G_{m}\left(s, t, h_{n}, h_{q}\right)$. Here $\hat{D}^{k}$ denotes the $k$ th order directional derivative as defined in [11]. It follows as in [10, p. 354] that $\alpha_{n, q}^{(k)}(r)=$ $\alpha_{q, n}^{(k)}(r)$ for $(n, q) \in Q_{k}$.

THEOREM 4.1. The following statements are equivalent.

a) $\alpha_{n, q}^{(k)}(r) \geq 0$ for all $(n, q) \in Q_{k}$.

b) If $p \in \mathcal{P}_{m}\left(\mathbb{R}^{2}\right)$ and if $\left|p\left(h_{n}, h_{q}\right)\right| \leq 1$ whenever $(n, q) \in Q_{k}$, then

$$
\left|\hat{D}^{k} p(r, r)(1,-1)\right| \leq p_{m}^{(k)}(r)
$$

It is shown in [11] that V. A. Markov's classical theorem and its extension to any real normed linear space is a consequence of Theorem 4.1. In fact, by [13], if $p_{m}=T_{m}$ and $(n, q) \in Q_{k}$, then $\alpha_{n, q}^{(k)}(r) \geq 0$ for $r \geq 1$ when $k$ is even and for $r \geq \cos \left(\frac{\pi}{2 m}\right)$ when $k$ is odd. Also, if $p_{m}=U_{m}$ and $(n, q) \in Q_{k}$, then $\alpha_{n, q}^{(k)}(r) \geq 0$ for $r \geq \cos \left(\frac{\pi}{m+2}\right)$ when $k$ is even and for $r \geq \cos \left(\frac{\pi}{2(m+2)}\right)$ when $k$ is odd. However, condition $(a)$ does not hold in many instances even for polynomials with degree 2 .

EXAMPLE 4.2. Let $m=k=2$ and $r \geq 0$. We show that condition (a) holds if and only if $a d=b c$. Thus, in particular, condition $(a)$ does not hold for Chebyshev polynomials of the third and fourth kinds. Put

$$
A=a d-b c, \quad B=a d+b c, \quad C=\sqrt{A^{2}+8 a c} .
$$

Then

$$
h_{0}=\frac{-B+C}{2 a c}, \quad h_{1}=-\frac{b}{a}, \quad h_{2}=\frac{-B-C}{2 a c}
$$


and

$$
\begin{aligned}
\alpha_{0,0}^{(2)}(r) & =\frac{a^{2} A(C-A)}{2 c^{2}}, & \alpha_{1,1}^{(2)}(r) & =\frac{2 a^{3}}{c}, \\
\alpha_{2,0}^{(2)}(r) & =\frac{4 a^{3}}{c}, & \alpha_{2,2}^{(2)}(r) & =-\frac{a^{2} A(C+A)}{2 c^{2}} .
\end{aligned}
$$

Thus (a) holds if and only $A=0$.

Proof of Theorem 4.1. Define a linear functional $\ell$ on $\mathcal{P}_{m}\left(\mathbb{R}^{2}\right)$ by

$$
\ell(p)=\hat{D}^{k} p(r, r)(1,-1)
$$

(a) $\Rightarrow$ (b). If $p \in \mathcal{P}_{m}\left(\mathbb{R}^{2}\right)$, then it follows from Theorem 3.2 that

$$
\ell(p)=\sum_{(n, q) \in Q_{k}} p\left(h_{n}, h_{q}\right) \ell\left(P_{n, q}\right)
$$

since $\ell\left(V_{j}\right)=0$ for $j=0, \ldots, m$ by formula (13) of [10]. In particular, taking $p(s, t)=p_{m}(s)$, we obtain

$$
p_{m}^{(k)}(r)=\sum_{(n, q) \in Q_{k}}(-1)^{n} \ell\left(P_{n, q}\right) .
$$

If $\left|p\left(h_{n}, h_{q}\right)\right| \leq 1$ for all $(n, q) \in Q_{k}$, then by the triangle inequality

$$
|\ell(p)| \leq \sum_{(n, q) \in Q_{k}}\left|\ell\left(P_{n, q}\right)\right|=p_{m}^{(k)}(r)
$$

since $\left|\ell\left(P_{n, q}\right)\right|=(-1)^{n} \ell\left(P_{n, q}\right)$ for $(n, q) \in Q_{k}$ by (a).

(b) $\Rightarrow\left(\right.$ a). Define $p(s, t)=p_{m}(s)-(-1)^{n_{0}} P_{n_{0}, q_{0}}(s, t)$, where $\left(n_{0}, q_{0}\right) \in Q_{k}$. Then $\left|p\left(h_{n}, h_{q}\right)\right| \leq 1$ for all $(n, q) \in Q_{k}$ so $\ell(p) \leq \ell\left(p_{m}\right)$ by (b). Thus (a) follows.

\section{APPENDIX: Interpolation and Cubature in $\mathbb{R}^{2}$}

The purpose of this appendix is to give the basic general facts we use that connect Lagrange polynomials, reproducing kernels and cubature formulas. It is based on ideas in [12]. (Another approach is given in [18].) See [6] and [8] for surveys of these extensive areas.

Given $m \geq 1$, let $\mathcal{P}_{m}\left(\mathbb{R}^{2}\right)$ denote the space of all real-valued polynomials in two variables with degree at most $m$. Let $\left\{x_{i}\right\}_{i=1}^{n}$ be $n$ distinct points in $\mathbb{R}^{2}$ and suppose $\left\{P_{i}\right\}_{i=1}^{n}$ is a corresponding set of Lagrange polynomials in $\mathcal{P}_{m}\left(\mathbb{R}^{2}\right)$.

Proposition A-1. Suppose $V_{1}, \ldots, V_{N}$ are linearly independent polynomials in $\mathcal{P}_{m}\left(\mathbb{R}^{2}\right)$ such that $V_{j}\left(x_{i}\right)=0$ for all $i=1, \ldots, n$ and $j=1, \ldots, N$. If $n+$ $N \geq \operatorname{dim} \mathcal{P}_{m}\left(\mathbb{R}^{2}\right)$ then for every $p \in \mathcal{P}_{m}\left(\mathbb{R}^{2}\right)$ there is a linear combination $\bar{p}$ of $V_{1}, \ldots, V_{N}$ such that

$$
p=\sum_{i=1}^{n} p\left(x_{i}\right) P_{i}+\bar{p} .
$$

Proof. Define a linear map $L: \mathcal{P}_{m}\left(\mathbb{R}^{2}\right) \rightarrow \mathbb{R}^{n}$ by $L(p)=\left(p\left(x_{1}\right), \ldots, p\left(x_{n}\right)\right)$. Since $\mathcal{P}_{m}\left(\mathbb{R}^{2}\right)$ contains Lagrange polynomials for $\left\{x_{i}\right\}_{i=1}^{n}$, the range of $L$ contains the standard basis for $\mathbb{R}^{n}$ so $\operatorname{Rank}(L)=n$. By hypothesis, $N \leq \operatorname{Nullity}(L)$. 
Since $\operatorname{Rank}(L)+\operatorname{Nullity}(L)=\operatorname{dim} \mathcal{P}_{m}\left(\mathbb{R}^{2}\right)$, it follows from the hypotheses that $\operatorname{Nullity}(L)=N$. Thus, $V_{1}, \ldots, V_{N}$ is a basis for the null space of $L$. $(\mathrm{A}-1)$.

Now let $\bar{p}=p-\sum_{i=1}^{n} p\left(x_{i}\right) P_{i}$. Then $\bar{p}$ is in the null space of $L$ so $p$ satisfies

Let $\mu$ be a positive measure on $\mathbb{R}$ and let $\mu \times \mu$ be the product measure on $\mathbb{R}^{2}$. Given a positive integer $m$, we suppose that

$$
(p, q)=\iint_{\mathbb{R}^{2}} p(s, t) q(s, t) d(\mu \times \mu)(s, t)
$$

defines an inner product on $\mathcal{P}_{m}\left(\mathbb{R}^{2}\right)$. For example, if $w(t)$ is a weight function (as defined in $[\mathbf{4}]$ ), then we may take $d \mu(t)=w(t) d t$ so that $(\cdot, \cdot)$ is an inner product with

$$
d(\mu \times \mu)(s, t)=w(s) w(t) d s d t .
$$

Let $\left\{p_{j}(t)\right\}_{j=0}^{\infty}$ be an orthonormal system of polynomials with respect to $\mu$ and let $\mathcal{S}_{m}$ be the subspace of $\mathcal{P}_{m}\left(\mathbb{R}^{2}\right)$ spanned by the polynomials $\phi_{j}(s, t)=$ $p_{m-j}(s) p_{j}(t), j=0, \ldots, m$. Let $K_{m-1}(x, y)$ be the reproducing kernel for $\mathcal{P}_{m-1}\left(\mathbb{R}^{2}\right)$ with respect the inner product (A-2) on this space.

Theorem A-2. Suppose $\left\{\alpha_{i}\right\}_{i=1}^{n}$ are real numbers. Conditions (a) and (b) below are equivalent.

a) If $p \in \mathcal{P}_{m}\left(\mathbb{R}^{2}\right)$ then there is an $S \in \mathcal{S}_{m}$ with

$$
p=\sum_{i=1}^{n} p\left(x_{i}\right) P_{i}+S
$$

Also, for each $i=1, \ldots, n$, there is an $S_{i} \in \mathcal{S}_{m}$ with

b)

$$
P_{i}(x)=\alpha_{i} K_{m-1}\left(x, x_{i}\right)+S_{i}(x), \quad x \in \mathbb{R}^{2} .
$$

$$
\iint_{\mathbb{R}^{2}} p(s, t) d(\mu \times \mu)(s, t)=\sum_{i=1}^{n} \alpha_{i} p\left(x_{i}\right)
$$

for all $p \in \mathcal{P}_{2 m-1}\left(\mathbb{R}^{2}\right)$.

Proof. (a) $\Rightarrow$ (b). Let $p_{1} \in \mathcal{P}_{m-1}\left(\mathbb{R}^{2}\right)$. For each $i=1, \ldots, n$, by the reproducing property,

$$
\alpha_{i} p_{1}\left(x_{i}\right)=\left(p_{1}, \alpha_{i} K_{m-1}\left(\cdot, x_{i}\right)\right)=\left(p_{1}, P_{i}-S_{i}\right)=\left(p_{1}, P_{i}\right)
$$

since $\left(p_{1}, S_{i}\right)=0$. Now if $p_{2} \in \mathcal{P}_{m}\left(\mathbb{R}^{2}\right)$, then it follows from (A-3) that

$$
\left(p_{1}, p_{2}\right)=\sum_{i=1}^{n} p_{2}\left(x_{i}\right)\left(p_{1}, P_{i}\right)+\left(p_{1}, S\right)=\sum_{i=1}^{n} \alpha_{i} p_{1}\left(x_{i}\right) p_{2}\left(x_{i}\right) .
$$

Therefore (A-5) holds when $p=p_{1} p_{2}$ and thus it holds when $p \in \mathcal{P}_{2 m-1}\left(\mathbb{R}^{2}\right)$ since $p$ is a linear combination of monomials of the form $p_{1} p_{2}$.

(b) $\Rightarrow$ (a). We first observe that if $q \in \mathcal{P}_{m}\left(\mathbb{R}^{2}\right)$ and if $\left(q, p_{1}\right)=0$ for all $p_{1} \in$ $\mathcal{P}_{m-1}\left(\mathbb{R}^{2}\right)$, then $q \in \mathcal{S}_{m}$. Indeed, by the orthogonal decomposition there exists a $p_{1} \in \mathcal{P}_{m-1}\left(\mathbb{R}^{2}\right)$ and an $S \in \mathcal{S}_{m}$ with $q=p_{1}+S$. Then $\left(p_{1}, p_{1}\right)=\left(p_{1}, p_{1}\right)+\left(S, p_{1}\right)=$ $\left(q, p_{1}\right)=0$ so $p_{1}=0$. 
Let $p \in \mathcal{P}_{m}\left(\mathbb{R}^{2}\right)$ and take $q=p-\sum_{i=1}^{n} p\left(x_{i}\right) P_{i}$. Clearly $q\left(x_{i}\right)=0$ whenever $i=1, \ldots, n$. If $p_{1} \in \mathcal{P}_{m-1}\left(\mathbb{R}^{2}\right)$ then $q p_{1} \in \mathcal{P}_{2 m-1}\left(\mathbb{R}^{2}\right)$ and so $\left(q, p_{1}\right)=0$ by $(\mathrm{A}-5)$. Hence $q \in \mathcal{S}_{m}$, which is $(\mathrm{A}-3)$.

Now, given $i=1, \ldots, n$, take $q(x)=P_{i}(x)-\alpha_{i} K_{m-1}\left(x, x_{i}\right)$. If $p_{1} \in \mathcal{P}_{m-1}\left(\mathbb{R}^{2}\right)$, then $\left(p_{1}, P_{i}\right)=\alpha_{i} p_{1}\left(x_{i}\right)$ by $(\mathrm{A}-5)$ and $\left(p_{1}, K_{m-1}\left(\cdot, x_{i}\right)\right)=p_{1}\left(x_{i}\right)$ by the reproducing property. Hence $\left(q, p_{1}\right)=\left(p_{1}, q\right)=0$ for all $p_{1} \in \mathcal{P}_{m-1}\left(\mathbb{R}^{2}\right)$ so $q \in \mathcal{S}_{m}$, as required.

Corollary A-3. (Compare [3].) Suppose $\left\{P_{i}\right\}$ satisfies $(A-3)$ and suppose $P_{i}(x)=\alpha_{i} G_{m}\left(x, x_{i}\right)$ for $i=1, \ldots, n$, where

$$
G_{m}(x, y)=K_{m-1}(x, y)+\sum_{j=0}^{m} \sum_{k=0}^{m} \beta_{j, k} \phi_{j}(x) \phi_{k}(y), \quad x, y \in \mathbb{R}^{2},
$$

with $\beta_{j, k}=\beta_{k, j}$ for $0 \leq j, k \leq m$. Then $p=\sum_{i=1}^{n} p\left(x_{i}\right) P_{i}$ for all $p \in \mathcal{P}_{m-1}\left(\mathbb{R}^{2}\right)$.

Proof. Note that the hypotheses of part (a) of Theorem A-2 are satisfied since (A-6) implies (A-4). Let $y \in \mathbb{R}^{2}$ and $p \in \mathcal{P}_{m-1}\left(\mathbb{R}^{2}\right)$. By the reproducing property, (A-6) and part (b), we have

$$
p(y)=\left(p, K_{m-1}(\cdot, y)\right)=\left(p, G_{m}(\cdot, y)\right)=\sum_{i=1}^{n} \alpha_{i} p\left(x_{i}\right) G_{m}\left(x_{i}, y\right) .
$$

Since $G_{m}$ is symmetric in $x$ and $y$, we have $\alpha_{i} G_{m}\left(x_{i}, y\right)=P_{i}(y)$ and the asserted identity follows.

\section{References}

1. G. Andrews, R. Askey and R. Roy, Special Functions, Encyclopedia of Mathematics and its Applications 71, Cambridge University Press, Cambridge, 1999.

2. R. Askey and J. Wilson, Some basic hypergeometric orthogonal polynomials that generalize Jacobi polynomials, Mem. Amer. Math. Soc. 54 (1985), no. 319.

3. B. Bojanov and G. Petrova, On minimal cubature formulae for product weight functions, J. Comput. Appl. Math. 85 (1997), 113-121.

4. T. S. Chihara, An Introduction to Orthogonal Polynomials, Math. and its Appl., Vol. 13 , Gordon and Breach, New York-London-Paris, 1978.

5. J. M. Cohen and A. R. Trenholme, Orthogonal polynomials with a constant recursion formula and an application to harmonic analysis, J. Funct. Anal. 59 (1984), 175-184.

6. R. Cools, Constructing cubature formulae: the science behind the art, Acta Numerica 6, 1997, $1-54$.

7. P. J. Davis and P. Rabinowitz, Methods of Numerical Integration, 2nd ed., Academic Press, New York, 1984.

8. M. Gasca and T. Sauer, Polynomial interpolation in several variables, Adv. Comput. Math. 12 (2000), no. 4, 377-410.

9. J. Geronimus, On a set of polynomials, Ann. of Math. (2) 31 (1930), 681-686.

10. L. A. Harris, Multivariate Markov polynomial inequalities and Chebyshev nodes, J. Math. Anal. Appl. 338 (2008), 350-357.

11. A proof of Markov's theorem for polynomials on Banach spaces, J. Math. Anal. Appl. 368 (2010), 374-381.

12. Bivariate Lagrange interpolation at the Chebyshev nodes, Proc. Amer. Math. Soc. 138 (2010), 4447-4453.

13. A bivariate Markov inequality for Chebyshev polynomials of the second kind, J. Approx. Th. 163 (2011), 1806-1814.

14. J. C. Mason and D. C. Handscomb, Chebyshev Polynomials, Chapmann \& Hall/CRC, Boca Raton, 2003. 
15. G. Monegato and A. Palamara Orsi, On a set of polynomials of Geronimus, Boll. Un. Mat. Ital. B (6) 4 (1985), 491-501.

16. N. Saitoh and H. Yoshida, The infinite divisibility and orthogonal polynomials with a constant recursion formula in free probability theory, Probab. Math. Statist. 21 (2001), 159-170.

17. T. Stoll, Decomposition of perturbed Chebyshev polynomials, J. Comput. Appl. Math. 214 (2008), 356-370.

18. Yuan Xu, Gaussian cubature and bivariate polynomial interpolation, Math. Comp. 59 (1992), $547-555$.

19. Quasi-orthogonal polynomials, quadrature, and interpolation, J. Math. Anal. Appl. 182 (1994), 779-799.

20. Lagrange interpolation on Chebyshev points of two variables, J. Approx. Theory 87 (1996), 220-238.

Department of Mathematics, University of Kentucky, Lexington, Kentucky 40506 E-mail address: larry@ms.uky.edu 